\title{
Présentation. Théories de la narration
}

\section{Pascale Mounier}

\section{(2) OpenEdition}

Journals

Édition électronique

URL : https://journals.openedition.org/rhetorique/1330

DOI : 10.4000/rhetorique.1330

ISSN : 2270-6909

\section{Éditeur}

UGA Éditions/Université Grenoble Alpes

\section{Édition imprimée}

ISBN : 978-2-37747-345-8

\section{Référence électronique}

Pascale Mounier, «Présentation. Théories de la narration », Exercices de rhétorique [En ligne], 18 | 2022, mis en ligne le 24 février 2022, consulté le 26 février 2022. URL : http://journals.openedition.org/ rhetorique/1330; DOI : https://doi.org/10.4000/rhetorique.1330

Ce document a été généré automatiquement le 26 février 2022.

\section{(c) (i) (2)}

Les contenus de la revue Exercices de rhétorique sont mis à disposition selon les termes de la Licence Creative Commons Attribution - Pas d'Utilisation Commerciale - Partage dans les Mêmes Conditions 4.0 International. 


\title{
Présentation. Théories de la narration
}

\author{
Pascale Mounier
}

1 Le terme narration, emprunté au latin narratio, n'a pas le même sens dans le champ de la rhétorique que dans celui de la littérature. D'un côté il s'agit de l'exposé du fait tel qu'il s'est passé ou qu'il a pu se passer. Cela concerne des individus réels. L'orateur use de la narration pour soutenir une cause ou réfuter une cause adverse. Cicéron rend compte par exemple dans le Pro Milone des agissements de Milon, assassin de Clodius, de façon à discréditer les accusations de méchanceté et de préméditation qui pèsent sur celui-ci ${ }^{1}$. De l'autre, la narration est l'acte de rapporter de façon détaillée les actions de personnages. L'auteur est amené à produire un texte plus ou moins long, selon le genre qu'il choisit, roman, nouvelle, fable ou épopée ${ }^{2}$. La narratio oratoire rend compte d'une chronologie dans la perspective d'une démonstration ${ }^{3}$. La narratio que l'on peut appeler " poétique ", «fabuleuse » ou encore " mimétique » invente les événements et produit une organisation, conforme ou non au déroulement chronologique, qui capte en soi l'attention.

2 Les deux types de narrations se prêtent en partie à une codification technique. Les traités de rhétorique d'une part et les traités de poétique et les études de narratologie d'autre part scrutent les mécanismes mobilisés. Or, quoique distinctes à l'origine, les traditions théoriques antiques et modernes approchant la notion de "narration » ne sont pas complétement indépendantes. Elles se croisent d'abord par leurs objets. Aristote cite par exemple dans la Rhétorique le récit d'Ulysse devant Alcinoos, aux chants IX à XII de l'Odyssée : le personnage rapporte le périple qu'il a effectué durant une vingtaine d'années en présentant ses aventures comme actuelles ${ }^{4}$. Joseph de Jouvancy mentionne quant à lui dans le Candidatus rhetoricce la fable « Le Loup et l'Agneau ", sûrement dans la version de Phèdre ${ }^{5}$. Les traités de rhétorique signalent même l'existence de narrations situées hors de la cause. L'auteur de la Rhetorica ad Herennium et Cicéron dans son De inventione indiquent trois catégories ( genera») de narrations ${ }^{6}$. La première porte sur la cause; la seconde, qui consiste à faire une digression en évoquant par exemple une autre action de la personne concernée ou 
d'autres actions semblables d'autres personnes, ne porte pas sur la cause mais entretient un rapport indirect avec celle-ci ; la troisième n'a aucun rapport avec les débats publics ${ }^{7}$. Ce dernier genre, exercice aussi agréable qu'utile, doit être pratiqué par l'orateur pour qu'il sache plus tard produire une narration dans le cadre d'un procès. La narration poétique a ainsi une place dans l'apprentissage de l'art oratoire. Les deux ensembles théoriques mobilisent ensuite à l'occasion des concepts similaires. Ils s'empruntent des notions, quitte à les rebaptiser et à en adapter le contenu à leurs propres cadres. La rhétorique latine suit ainsi la rhétorique hellénistique, qui connaît elle-même la Poétique d'Aristote. On trouve en effet des éléments de la pensée aristotélicienne de l'intrigue dans les passages que la Rhetorica ad Herennium et le De inventione consacrent à la narration. Les traités et les commentaires du $\mathrm{xvI}^{\mathrm{e}}$ siècle évoquant un genre comme l'épopée acclimatent de leur côté des catégories rhétoriques. Des points de passage entre le domaine oratoire et le champ poétique existent bel et bien. Ils méritent d'être identifiés et analysés pour comprendre ce qui anime l'orateur et le poète quand ils font une narration et pour voir autant la spécificité que la différence de leurs méthodes.

3 Cela implique d'abord d'enquêter sur les éléments de théorie livrés par les traités et les manuels produits $d u \mathrm{XV}^{\mathrm{e}}$ au XVIII ${ }^{\mathrm{e}}$ siècle. L'apport conceptuel de la période gagne à être mis en perspective par rapport à l'amont, la codification antique, et à l'aval, les théories actuelles du récit. Il est ensuite intéressant de prendre en compte des réalisations pratiques. Le cas du récit inséré dans une œuvre narrative de fiction constitue en l'occurrence un bon exemple des usages littéraires de la narration oratoire.

\section{Théories de la narration}

4 La partie "Atelier " de ce numéro fournit une très ample matière pour saisir la codification de la narratio livrée par les traités. Lise Charles et Suzanne Duval proposent une édition et une traduction d'extraits du commentaire d'Antonio De' Conti, dit Majoragius, des Partitiones oratorice de Cicéron ${ }^{8}$. Elles font sortir de l'oubli le professeur de rhétorique milanais, dont elles retracent la carrière dans l'introduction de leur édition. Jean Lecointe identifie quant à lui, dans son article « Matériaux pour l'étude de la théorie de la narration dans la rhétorique humaniste ", bon nombre de passages, rédigés en latin ou en français, consacrés à la narration aux XVe, $\mathrm{XVI}^{\mathrm{e}}$ et $\mathrm{XVII}{ }^{\mathrm{e}}$ siècles. Il résume l'apport de chacun d'eux et indique le cas échéant d'où peut provenir telle ou telle idée quand elle apparaît pour la première fois. Il organise sa liste en sept rubriques, correspondant aux grands domaines dans lesquels se développe la réflexion théorique, et adopte une logique chronologique à l'intérieur de celles-ci. Un tel relevé analytique fait émerger des concepts et des cadres de classification. Tâchons de les identifier ${ }^{9}$. Nous allons ainsi repérer les éléments de définition propres respectivement à la narration oratoire et à la narration poétique puis identifier les zones de contact entre l'une et l'autre. On pourra se reporter au recueil de traités commenté de Jean Lecointe pour avoir des précisions sur les ouvrages que nous mentionnons.

\section{a. Narration oratoire}

5 La narration oratoire apparaît seulement dans les traités de rhétorique. Elle fait l'objet non seulement des ouvrages généraux de tradition antique, qui diffusent les catégories 
des Anciens en les reprenant à l'identique ou en les adaptant, mais aussi des manuels scolaires, qui offrent des exercices préparatoires aux élèves des classes de rhétorique. On l'aborde ainsi alternativement, même si c'est éventuellement dans le même traité, soit dans sa forme sophistiquée soit dans son mode élémentaire ${ }^{10}$. La doctrine la plus complète se trouve dans le premier massif, apparu historiquement avant le second.

La narratio est envisagée, au sein des traités généraux, dans le cadre de la dispositio. C'est une des étapes du discours de l'orateur, qui se situe entre l'exorde et la discussion, autrement dit avant la réfutation et la confirmation. Elle a une importance particulière dans le genre judiciaire puisque l'avocat ou l'accusé veulent établir et interpréter des faits. Le prêtre peut aussi la convoquer dans le sermon. La matière exposée au barreau ou en chaire n'est en l'occurrence pas nécessairement conforme à ce qui s'est passé. Selon Jean-Baptiste Crevier, la « Narration oratoire " peut modifier le vrai pour servir la cause, tandis que la «Narration historique » s'en tient au vrai ${ }^{11}$. L'orateur traite par ailleurs tout ou partiellement le fait. Il peut présenter les individus, le moment et l'endroit de l'action et préciser la façon dont celle-ci s'est déroulée. Les traités fournissent souvent une liste de ces composantes. René Bary y voit des "parties»: «Les parties de la narration, sont les personnes, le temps, le lieu, l'action, le moyen, la maniere, et la fin ${ }^{12}$ ». Jacques Du Roure parle quant à lui de «circonstances » et donne le "vers" qui permet de les retenir: "Qui? quoy? par quelle cause? où? quand? pourquoy? comment ${ }^{13}$ ?». Les auteurs les plus prolixes s'adonnent à partir de là à la création de typologies plus ou moins fournies. Bartolomeo Cavalcanti propose différentes narrations selon les genres rhétoriques et les manières de servir la cause ${ }^{14}$. Nicolas Caussin identifie de nombreuses espèces de narrations pouvant être mobilisées dans le genre judiciaire ${ }^{15}$. Lise Charles et Suzanne Duval constatent qu'il reprend en fait littéralement dans les chapitres sur la narration de ses De eloquentice sacrce et humance parallela presque toute la théorie forgée par Majoragius dans son commentaire des Partitiones oratorice. Elles éditent et traduisent les passages repris et les quelques ajouts faits par Caussin.

7 Tous les traités pointent les virtutes ou qualités de la narration. Les trois qualités canoniques sont au fond celles de tout discours oratoire. Comme le montre l'étymologie du mot, la narration implique un partage de savoir, une volonté de comprendre et de faire comprendre ${ }^{16}$. Elle cherche à déterminer des responsabilités humaines ${ }^{17}$. Il s'agit en l'occurrence de mettre le juge en état de comprendre, de retenir et de croire ce qu'on lui dit. Les règles en sont établies et commentées dès l'Antiquité, comme le montre Jean Lecointe dans son article «L'agrément des faits : le retour de la "vertu" de suauitas dans la rhétorique humaniste de la narration (1450-1710)». La narration doit être brève («brevis »), claire ("dilucida», «aperta ») et vraisemblable («probabilis", "veri similis», «credibilis») ${ }^{18}$. La brièveté consiste essentiellement à sélectionner les éléments qui servent la cause et à ne pas se répéter. Cicéron insiste dans le De inventione sur cet aspect puis limite son poids dans les traités suivants ${ }^{19}$. Quintilien pose ouvertement la question du rapport entre la brièveté et la longueur : il indique qu'il se peut que la narration étendue soit brève et qu'inversement une narration courte ne soit pas brève, ce qu'il condamne ${ }^{20}$. La clarté se traduit surtout par le suivi de la chronologie des événements, l'évitement des digressions et une expression compréhensible. La vraisemblance tient à la possibilité de réalisation de ce qui est dit, au rapport entre les motifs et les personnes ainsi qu'au respect des bienséances ${ }^{21}$. 
Une quatrième virtus s'ajoute aux trois premières, l'agrément (« suavitas »). On la trouve régulièrement à partir du début $d u \mathrm{XVII}^{\mathrm{e}}$ siècle, par exemple Gerardus Joannes Vossius et Charles Pajot, et avant cela dans quelques traités, comme ceux de Louis de Grenade et Lodovico Carbone. La qualité renvoie à des réalités assez disparates selon les auteurs, qui concernent aussi bien le domaine de l'invention que celui de l'élocution. Après avoir indiqué pour qualités de la narration «Clarté Vray-semblance Agrément Breveté », Du Roure précise ainsi qu'il entend par agrément « les choses extraordinaires, ou du moins celles que l'on dit d'une façon extraordinaire ${ }^{22} »$. Cette suavitas est apparue pour la première fois dans la rhétorique latine avec les Partitiones oratorice, donc tardivement chez Cicéron ${ }^{23}$. Il faut pour Cicéron non seulement choisir des mots doux et sonores, faire des répétitions ou suspendre l'avancée du discours, toutes choses contradictoires avec la clarté remarquons-le, mais aussi mettre en avant des faits qui étonnent ("admirationes») ou qui provoquent de l'attente ("expectationes»), des dénouements inattendus, des dialogues, de la douleur, de la colère, de l'allégresse, etc. ${ }^{24}$. La liste des faits extraordinaires captivants est très certainement reprise à la courte théorie touchant la narration qui porte sur les personnes de la Rhetorica ad Herennium et du De inventione, un des deux types de narrations poétiques ${ }^{25}$. Selon ces deux traités, celle-ci doit mettre en œuvre la festivitas en présentant des événements variés et des sentiments divers, comme la gravité, la douceur, l'espoir et la dissimulation, et un cours des événements saisissant, par exemple un revirement complet du sort ou une fin heureuse. Cette prise en compte des effets de l'inventio sur l'auditeur-lecteur hérite très indirectement de la Poétique d'Aristote et entre en résonance au $\mathrm{xvI}^{\mathrm{e}}$ siècle avec l'émergence de la notion de « suspens ${ }^{26}$ ». La narration oratoire se voit donc moins liée à la mise au jour des antécédents, des circonstances et des conséquents du fait que préoccupée du plaisir qui capture l'âme, même s'il contribue à persuader ${ }^{27}$. Elle subit l'influence de procédés propres à la mimèsis.

\section{b. Narration poétique}

9 La narration poétique est évoquée prioritairement dans le cadre de la théorie poétique antique et moderne de l'intrigue et de la narratologie actuelle. Elle apparaît aussi dans la réflexion rhétorique. Les traités généraux, depuis la Rhetorica ad Herennium et le De inventione, l'envisagent, comme nous avons vu, comme une étape nécessaire pour se former à la pratique du débat public. Les progymnasmata de leur côté la font pratiquer aux élèves en vue de les préparer aux classes de rhétorique. Ces manuels scolaires, qui héritent de l'enseignement oral des rhéteurs grecs tout en standardisant la doctrine de Quintilien, sont apparus à la fin du $\mathrm{I}^{\mathrm{er}}$ siècle, notamment à l'initiative d'Aélius Théon, du Pseudo-Hermogène et d'Aphtonios. Ils sont très connus au xvi ${ }^{e}$ siècle : ceux du PseudoHermogène et d'Aphtonios ont été traduis en latin au Moyen Âge et celui de Théon est traduit en latin en 1541. Les collections d'exercices donnent une place privilégiée à la narration poétique, très facile à proposer car elle n'entre pas dans un discours à visée persuasive.

Toutes les théories tendent à établir une distinction entre la narration fictive, donc poétique, et la narration vraie. L'opposition entre poésie et histoire remonte à Aristote. La Poétique réévalue, contre Platon, le faux par rapport au vrai sur la base de la mimèsis, c'est-à-dire de l'art de produire une image cohérente du réel. Lucien de Samosate reproche de son côté aux poètes de se prétendre historiens. La tradition rhétorique 
défend aussi l'histoire au moyen de l'idée de Cicéron, reprise par Quintilien, selon laquelle celle-ci est « magistra vito ${ }^{28}$ ». Elle apporte par ailleurs des outils pour penser la frontière entre le vrai et le faux. L'auteur de la Rhetorica ad Herennium et Cicéron dans le De inventione mettent en effet en place, quoique rapidement, la triade fabula, historia et argumentum ${ }^{29}$. Dans leur raisonnement il s'agit de décliner le contenu, les partes, d'une sous-catégorie de la narration poétique et d'y associer des genres. La narration qui ne touche pas l'éloquence a en l'occurrence pour sujet soit les faits («negotia»), soit les personnes («personce»). La narration des faits se décompose en trois espèces. La fabula, ni vraie ni vraisemblable, est illustrée par la tragédie ; l'historia, vraie, qui rapporte des faits situés ou non dans le siècle de celui qui écrit, est le propre de l'historien; l'argumentum, vraisemblable, est réalisé dans les comédies. L'approche du vraisemblable vient indirectement de la Poétique d'Aristote, qu'adapte le modèle commun des deux traités. Les ouvrages d'art oratoire modernes tendent à ne parler que des trois types, autrement dit à passer sous silence la catégorie englobante de la narration poétique ${ }^{30}$. Bary ne distingue ainsi pas entre narration concernant des faits et narration touchant des personnes. Il écrit simplement : «La matiere de la narration est vraye, possible ou fabuleuse ${ }^{31}$ ». Il va même jusqu'à improviser une typologie à partir de la triade latine en pointant cinq sortes de narrations, "judiciaire", "digressive", "fabuleuse ", " romanesque » et " prophetique ${ }^{32}$ ». Du Roure, économe en catégories, s'en tient quant à lui à deux espèces de narrations, les « fabuleuses » et les « veritables ${ }^{33}$ ». La rhétorique oppose donc les narrations plaisantes et les récits historiques mais contribue à la théorie des premières en les subdivisant en fonction de leur rapport au vrai. Avec la philosophie, elle loue certaines fables des poètes au motif que celles-ci contiennent un sens caché. Parmi les thuriféraires d'une interprétation allégorique de la narratio fabulosa se trouvent, à la suite de Macrobe dans le Commentarium in Ciceronis somnium Scipionis, Érasme dans le De duplici copia verborum ac rerum et Philipp Melanchthon dans son De Rhetorica libri tres.

Les traités de poétique réfléchissent au statut de la narration au sein de la poésie. Ils héritent lointainement des notions de mimèsis et de diégèsis codifiées par Platon et Aristote, qui désignent selon les cas la mise en intrigue dans son ensemble, ce que l'époque appelle fable, et des espèces de l'art littéraire, autrement dit des genres. On sait que pour Aristote dans la Poétique tout est mimèsis. La mimèsis peut être narrative, par exemple dans l'épopée, ou dramatique, au théâtre; dans tous les cas il faut développer une intrigue ("diégèsis »). Pour Platon, il y a trois genres, que Genette surnomme modes, liés au positionnement énonciatif du poète. La mimèsis, qui se définit par la prise de parole des personnages, se décompose en mode dramatique pur, réalisé dans la tragédie, et en mode mixte, illustré par l'épopée. La diégèsis renvoie au récit assumé par le seul poète ; le dithyrambe est ainsi un poème narratif non mimétique. La Renaissance retient de cette classification, hétérogène dans ses concepts mais uniforme sur le fond, trois genres. Le grammairien Diomède a forgé, au IV siècle, les expressions genus imitativum ou dramaticum, genus ennarrativum ou enunciativum et genus mixtum. Giovanni Francesco Stoa et François Dubois pointent à sa suite trois genres de poésie, le genus narrativum, le genus representativum et le genus medium ${ }^{34}$. La série tend dans les faits à se réduire à deux termes, le mode narratif intégrant presque toujours des paroles de personnages. Sa postérité dépasse l'époque moderne. Genette, en particulier, en fait un critère pour définir les grands genres.

12 Les exercices préparatoires proposés dans les classes apprennent plutôt à situer la narration poétique par rapport à la narration oratoire. Les collections comprennent 
traditionnellement une dizaine de formes de discours, qui ont peu varié depuis la période hellénistique, et en fournissent de multiples exemples. La première est la fable ( "mythos »), la seconde la narration (" diégèma »), la troisième l'anecdote ou la chrie ( chreia »), la quatrième la maxime («gnomon»), etc. Les trois premières ont un rapport avec le récit. La narration est la plus englobante, alors que la fable prend la forme d'un court récit à visée morale et que la troisième, quand elle a pour sujet l'action, se présente comme un récit développant les motifs émotionnels ou moraux et convoquant des citations d'autorités ou d'autres $\operatorname{cas}^{35}$. Elle est envisagée ici dans le cadre scolaire, non comme partie du discours, même si les trois ou quatre qualités de la tradition cicéronienne sont reprises. Au début du livre II de l'Institutio oratoria, Quintilien préconise en l'occurrence, alors qu'il réfléchit à la formation de l'orateur, de commencer à exercer les enfants à la rhétorique en leur faisant pratiquer la narration ${ }^{36}$. Il indique trois "genera » de narrations, la fabula, sujet des tragédies et des poèmes, autrement dit probablement des épopées, l'argumentum, matière des comédies, et l'historia, qu'il définit comme l'exposition d'un fait et comme gagnant à être vraie. Il semble adapter ici la triade de la narration des faits, qui est, rappelons-le, une des formes des narrations ne servant pas la cause, en indiquant que les deux premiers types sont du ressort du grammairien et que le troisième revient plus particulièrement au maître de rhétorique. En d'autres termes, dans les petites classes les élèves doivent écrire des récits en partant du mythe ou de situations de la vie courante; dans les grandes il leur faut se fonder sur des grands passages de l'histoire. Quintilien précise que l'élève en rhétorique gagne à s'adonner à la narration historique et demande de veiller à ne pas être trop abondant au plan du style, tout en autorisant à ce qu'il le soit au début. La typologie que forge un rhéteur comme Aphtonios, au IV siècle, est plus opératoire encore. Elle donne pour qualificatifs à la narratio les adjectifs poetica, historica et civilis, ce qui revient à rapprocher et à distinguer en même temps la poésie, l'histoire et la rhétorique. La narratio poétique se voit identifiée en tant que telle et la narratio oratoire détachée du récit historique.

Reinhard Lorich s'arrête longuement sur la narration dans son commentaire de la traduction des Progymnasmata d'Aphtonios par Rodolpho Agricola ${ }^{37}$. Il donne pour exemple de narration poétique un ample extrait des Métamorphoses d'Ovide, à savoir l'histoire de Pyrame et Thisbé. Aphtonios identifie lui-même comme quatrième qualité des trois sortes de narrations la "verborum proprietas", selon les termes d'Agricola, ce qui prouve une attention de sa part au style. Les traités de rhétorique des XVII et $\mathrm{XVIII}^{\mathrm{e}}$ siècles, quand ils ne sont pas purement et simplement des manuels, reprennent cette triade scolaire. François Pomey organise la section «De Narratione» de son Candidatus Rhetorica en suivant les Progymnasmata d'Aphtonios et traite successivement de la narratio poetica, de la narratio historica et de la narratio civilis ${ }^{38}$. Jouvancy, qui souhaite aussi servir les Jésuites dans leur enseignement de l'art oratoire, adapte ce manuel en lui ajoutant quatre parties contenant la doctrine habituelle des traités de rhétorique. Il mentionne la narration dans la partie II, consacrée à la disposition, mais ne la développe pas. Il réserve ses distinctions et ses exemples à la partie $\mathrm{V}$, qui compile les exercices dans la tradition d'Aphtonios. II fait le choix de rassembler la fable et la narration dans une même section, intitulée «De Fabula », ce qui laisse entendre qu'il y voit un même exercice ${ }^{39}$. Mais il indique en même temps que la narration est une sorte de genre ("quasi genus») englobant la fable et l'histoire. Il insiste en outre sur la suavitas parmi les quatre qualités et décline des « ornamenta " possibles de la narration, à savoir l'incise, le présent de narration et l'infinitif ainsi que des figures comme 
l'hypotypose, la suspension et l'exclamation. Ce Candidatus Rhetorica a dès lors une orientation poétique marquée. Un théoricien comme Érasme va quant à lui jusqu'à proposer dans son De duplici copia des séries de sujets et de genres susceptibles de faire l'objet de fictoe narrationes ${ }^{40}$. Le domaine de la narration en vient ainsi à regrouper, aussi bien dans la tradition théorique et la pratique scolaire de la rhétorique que dans la réflexion poétique, deux ensembles distincts, la narration fictive, traitée par la poésie, et la narration vraie, considérée ici comme propre à l'histoire et à la rhétorique ${ }^{41}$.

L'agencement de la narration est presque exclusivement attaché à la narration poétique. La poétique et la narratologie se préoccupent de la mise en intrigue et de la mise en récit, alors que l'art oratoire s'intéresse avant tout au contenu de l'intrigue. La narratologie structuraliste, forgée notamment par Propp, Lévi-Strauss, Greimas et Brémond, a tâché de scruter la "morphologie ", la "sémantique » et la "logique » des récits. Elle a mis en évidence le rôle du choix de tel ou tel événement pour le début, le nœud ou la fin, de la manière de rapporter la succession des actions, du calibrage de la longueur du récit, du fait de construire un schéma narratif simple ou au contraire complexe, etc. La narratologie post-structuraliste, qui trouve ses meilleurs représentants dans Genette, Ricœur et Baroni, refuse d'envisager le texte de façon autonome et insiste sur les effets du récit sur le lecteur. Elle prend en compte la « mise en intrigue ", c'est-à-dire les composantes, par exemple le nœud ou la fin, du récit, et la "tension narrative », décelant les émotions que suscite l'avancée du récit vers la fin ${ }^{42}$. L'ordre d'ensemble de la narration peut ainsi être ou ne pas être linéaire : dans la lignée d'Horace, les poéticiens distinguent l'ordo artificialis ou poeticus de l'ordo naturalis. Le récit peut commencer brutalement ou non : à la suite d'Horace à nouveau, Dubois et d'autres mesurent les avantages respectifs du début $a b$ ovo et du commencement in medias res. Les traités rhétoriques, qui sont sensibles à leur façon à la composition, parlent au contraire peu de l'ordre de la narration oratoire. Ils reprennent le cas échéant les catégories poétiques ${ }^{43}$. Mais ils donnent aussi des conseils spécifiques, par exemple sur le fait que les historiens font une narration continue alors que les orateurs interrompent l'exposé des faits par des remarques ${ }^{44}$.

La narration poétique gagne de l'autonomie par rapport au champ rhétorique à la fin de la période. Elle est peu à peu envisagée comme une forme littéraire en soi, qui se décline dans plusieurs sortes de récits, possède ses propres mécanismes et cultive ses propres effets. Dans ses Éléments de littérature en 1787, Jean-François Marmontel mobilise certes les distinctions forgées au départ dans un cadre oratoire, comme les catégories « exposé des faits » et « exposé des choses » et les qualités canoniques, mais procède surtout à l'élaboration d'une typologie fine des genres totalement ou partiellement narratifs, dans laquelle la narration oratoire et la narration historique ne sont que des espèces parmi beaucoup d'autres ${ }^{45}$.

\section{c. Zones de contact}

Les discours réflexifs se croisent à l'époque, nous venons de le voir. Mais la narratio oratoire et la narratio poétique ne se superposent pas : elles restent distinctes. Plutôt que leurs sujets, le vrai ou le faux, ce sont surtout leurs finalités, comme le fait d'étayer une démonstration ou de raconter pour faire naître le suspens en cultivant les détours ou pour faire réfléchir en cachant un sens, qui diffèrent. Cicéron prévenait déjà qu'on pouvait se passer de la seconde partie du discours si elle nuisait à la cause ${ }^{46}$. Mais il est des cas où l'orateur et l'écrivain peuvent se rapprocher dans leur pratique de la 
narration. Voyons cette proximité de moyens que les rhétoriciens et les poéticiens ne mentionnent pas explicitement mais que les catégories qu'ils manipulent permettent d'entrevoir.

L'orateur peut user de procédés poétiques, c'est-à-dire procurant du plaisir, dans son exposé du fait. Il rend par exemple l'action sensible en la mettant sous les yeux des auditeurs, selon le principe de l'evidentia, elle-même proche de l'enargeia ${ }^{47}$. Il cherche aussi l'agrément en cultivant les émotions et l'intérêt pour le cours des événements, ce qui se rapproche de la tension narrative. Quintilien indique déjà que des faits peuvent susciter des pathè, à savoir l'indignation ou la pitié, même si les émotions restent limitées ${ }^{48}$. Georges de Trébizonde insiste sur ce dosage des affects ${ }^{49}$. Il envisage des exceptions à la modération de la part de l'orateur, en particulier dans les mouvements véhéments, qu'il appelle procursus. Crevier signale aussi le cas des causes importantes et présentant une matière variée ${ }^{50}$. Il précise que, même si elle a tendance à être sobre, la prose peut être marquée à l'occasion par des « exclamations subites» ou «de violentes apostrophes ${ }^{51} »$. Les manuels scolaires regorgent d'ailleurs d'extraits de narrations oratoires ou historiques illustrant alternativement le style simple, le style moyen et le style élevé.

L'écrivain peut se soumettre de son côté à des exigences rhétoriques quand il compose un roman, une nouvelle, une fable ou une épopée. Il cultive alors la brièveté, évitant les détails ou les digressions. Il réduit les effets de vivacité, qui mobilisent les passions. Il recourt aux ornements simples. Les progymnasmata donnent ainsi la même fable écrite en langage simple puis en style orné. L'œuvre narrative ou l'extrait de récit tendent en outre parfois à démontrer une thèse.

Tout est bien sûr affaire de proportion. La narration oratoire tend par nature à limiter l'illusion référentielle, l'exhibition de la mise en récit, la présence du narrateur et l'amplification. La narration poétique évite, elle, de prendre pour matière des faits vrais et de mettre en avant la construction d'un savoir au moyen de la restitution de ceux-ci. Toujours est-il que la poéticité potentielle de la narration oratoire fait que certains traités font l'impasse à son sujet. Jean Oudart de Richesource élimine la narration quand il aborde la disposition ${ }^{52}$. Étienne Dubois de Bretteville préconise de la faire «la plus courte que l'on pourra ${ }^{53}$ ». Il n'y a donc pas que la rhétorique ramiste, qui privilégie l'elocutio et laisse de côté la dispositio, qui se méfie de la narration pour son caractère mêlé. Les spécialistes de l'éloquence juridique et de la prédication posent aussi régulièrement des bornes à son emploi au XVII ${ }^{\mathrm{e}}$ siècle.

\section{Récits insérés dans des fictions narratives}

La partie « Dossier » et la partie « Analyse d'un discours » du numéro illustrent une des zones de contact, à savoir le cas où une fiction produite entre le $\mathrm{Xv}^{\mathrm{e}}$ et le $\mathrm{XVIII}$ siècle intègre une narration mobilisant des dispositifs rhétoriques. Certaines séquences textuelles assumées par un personnage ont de fait pour but plus ou moins explicite de soutenir une démonstration. Le protagoniste rapporte les événements, qui peuvent appartenir ou non à l'action principale, en construisant une approche orientée des caractères, des intentions et du discours des protagonistes. Il construit aussi un point de vue dans sa manière de mettre en relation les faits. Il s'agit ainsi pour les contributeurs de déceler les modalités et les enjeux de la production d'une narration intelligible, efficace et intéressante au sein d'une œuvre mimétique. Pour arriver à 
dégager des procédures spécifiques malgré le large empan chronologique retenu, l'enquête qu'ils mènent se limite aux fictions narratives.

21 La narration à visée judiciaire répond parfaitement aux prescriptions des traités et des manuels. Marie-Gabrielle Lallemand s'arrête sur le premier récit inséré d'Artamene ou le Grand Cyrus. Alors que l'œuvre est un "long roman", les Scudéry s'efforcent d'être brefs ici. Imitant les tribunaux d'amour, ils ne décrivent pas les lieux, mobilisent peu de personnages, limitent les informations sur ceux-ci et offrent peu de discours directs. Ce phénomène exceptionnel montre par contraste la liberté que prennent les auteurs de narrations héroïques et galantes : si les « Histoire[s]» qui ont une dimension politique conservent un enjeu argumentatif par leur rapport avec l'action principale dans les romans de ce type, celles qui sont purement galantes ne servent aucune cause, leur intérêt étant de satisfaire la curiosité du lecteur pour la psychologie des personnages.

Le genre du pèlerinage allégorique peut intégrer pour sa part des récits secondaires, continus ou non, et leur assigner indirectement des fins délibératives ou épidictiques similaires à celles du récit principal. Dans Le Séjour d'Honneur d'Octavien de Saint-Gelais, examiné par Ellen Delvallée, les quatorze séquences narratives prises en charge par des personnages, L'Acteur compris, servent de façon indirecte le projet d'éloge du roi et de mise en garde contre les dangers de la vie courtisane. Les narrationes en question, qui ont un sujet historique, voire pseudo-autobiographique, ou mythique, sont parfois assumées par des protagonistes immoraux, comme Peu d'Avis ou Cas Fatal. Ellen Delvallée suggère que, pour éviter que le lecteur ne se laisse séduire par la virtuosité rhétorique de tels discours, O. de Saint-Gelais déjoue les attentes d'une bonne narratio en faisant entorse à la clarté, à la brièveté et à la vraisemblance.

Le roman, qui laisse aussi à l'occasion la parole à des personnages conteurs, suscite des rapports plus variés entre les niveaux de narration et entre les visées, esthétiques ou idéologiques, respectives de ceux-ci. Isabelle Delage-Béland s'intéresse ainsi à la narration prêtée à Maguelonne dans la version de Pierre de Provence réalisée par Jean Castilhon en 1770. Ce récit inventé se fonde sur la matière du roman idyllique du $\mathrm{Xv}^{\mathrm{e}}$ siècle : l'héroïne rapporte les aventures qu'elle a vécues pendant sa séparation de Pierre. La substitution d'un récit inséré dans un discours à la technique de l'entrelacement de deux fils narratifs permet de captiver le lecteur. Celui-ci apprend en même temps que Pierre et d'autres personnages des faits énoncés de façon non seulement brève et claire mais aussi sincère. L'ethos de la narratrice et quelques discours directs, inventés aussi, mettent en avant deux vertus centrales dans l'œuvre, la loyauté et la charité.

Mathieu Goux analyse de son côté le récit de la lettre XIX du roman épistolaire monologique Lettres de la Marquise de $M^{* * *}$ au Comte de $R^{* * *}$ de Crébillon. La Marquise rapporte au Comte, qu'elle aime, une soirée mondaine ennuyeuse à laquelle elle a participé la veille. Elle s'ingénie à accumuler les détails inutiles et à omettre les éléments nécessaires à l'information du destinataire et à la formulation d'une excuse auprès de celui-ci. L'infraction aux qualités de la lettre narrative peut s'interpréter comme une opération de l'auteur pour détacher le conte libertin de la vraisemblance et de la persuasion et en faire ostensiblement une fiction satirique, offrant un portrait déformant de la société noble du temps.

Les nouvelles insérées dans un recueil à cadre à la façon boccacienne ou de discours bigarrés sont quant à elles intéressantes en ce qu'elles prennent de l'autonomie par rapport au récit principal. Comment les lois de l'argumentation s'insinuent-elles dans 
les récits des devisants, qui respectent certes la brièveté, comme les narrations judiciaires, mais qui semblent s'éloigner d'un éventuel projet de démonstration assumé par l'auteur? C'est ce qu'examinent Véronique Montagne et Amina Houara pour des sous-genres différents de la nouvelle du $\mathrm{xVI}^{\mathrm{e}}$ siècle. Véronique Montagne considère d'emblée L'Heptameron de Marguerite de Navarre comme une collection de narrationes mises au service d'une idéologie forte. Mais elle affronte une objection possible à cette position de principe, à savoir la prolixité et l'artifice stylistique qui caractérisent les discours rapportés dans le cadre des nouvelles : si les personnages à qui les conteurs laissent la parole parlent généralement en respectant les trois qualités habituelles de la narration oratoire, certaines interventions construisent des raisonnements plus ou moins complexes et mobilisent tous les arguments possibles du genre délibératif. Or Véronique Montagne montre que les discours amples ne sont jamais vains: ils développent les convictions du devisant-conteur, ce qui fait qu'ils gardent une fonction argumentative.

Amina Houara envisage de son côté les Propos rustiques de Noël Du Fail. Des paysans y dialoguent joyeusement, citant des cas illustres, faisant de bons mots et rapportant des récits empruntés à la tradition de la nouvelle. Il semble que les interlocuteurs mobilisent un procédé au pouvoir immersif, qui sollicite donc l'imaginaire, consistant à mettre les faits évoqués sous les yeux de l'auditoire: l'evidentia. Les protagonistes projettent les auditeurs dans l'univers de la fiction en construisant une deixis spatiale, au moyen essentiellement de démonstratifs et d'adverbes de lieu. De son côté, le narrateur-auteur, Du Fail lui-même, s'attache à faire voir le monde rural. Amina Houara fait l'hypothèse que les narrations encadrées et le récit-cadre s'appuient sur cette poétique de l'évidence pour servir une même démonstration des vertus d'un âge d'or rustique révolu.

Les six contributions révèlent ainsi la diversité des situations qui présentent un récit inséré dans une fiction narrative. Elles témoignent plus largement du caractère fécond de l'étude de l'influence des principes de la narration oratoire sur la création littéraire.

\section{NOTES}

1. Voir un passage de la défense écrite par Cicéron, qui n'a finalement jamais été plaidée, traduit par Le Gras dans La Rhetorique Françoise, éd. Paris, [s. n.], 1671, II, 3, « De la narration », p. 119 : «Milon que l'on accuse d'avoir dressé sur le chemin l'appareil d'un meurtre, passa sur ce mesme chemin dans son carrosse avec sa femme, accompagné du foible secours de ses enfans, de ses servantes, et de son train ordinaire. ». Cicéron donne des éléments montrant l'insouciance de l'individu et de ses proches, ce qui récuse l'idée d'une volonté de Milon de se mettre en route pour tuer Clodius.

2. Genette distingue la « narration », l'acte narratif, du « récit », le résultat de la narration, et de l'« histoire », le contenu du récit. Une même histoire peut ainsi donner lieu à un récit condensé ou à un récit développé. La langue courante emploie pour sa part les substantifs narration, récit et histoire comme des synonymes, au sens large de «relation détaillée, écrite ou orale, d'un événement ». 
3. Voir la réflexion que mène $\mathrm{Ch}$. Noille dans « Narratio / narration? La rhétorique et la langue française ", in Les Intraduisibles du vocabulaire critique (XVI ${ }^{e}-X V I I I^{e}$ siècle), dir. C. Barbafieri, D. Denis et L. Susini, Littératures classiques, $n^{\circ} 96 / 2,2018$, p. 85-97, sur ce qu'implique le fait de recourir à une narration pour un orateur. La critique propose de traduire le terme narratio en contexte rhétorique par « dispositif narratif » (p. 97). Elle voit dans la narration oratoire une "mise en débat du factuel, orientée, ordonnée et enchaînée par la seule visée argumentative » (p. 92).

4. Rhét., III, 16.

5. Candidatus rhetoriç, éd. Paris, Jean Barbou, 1714, V, « De fabula », p. 180 (L'Élève de rhétorique, éd. et trad. D. Denis et F. Goyet, Paris, Classiques Garnier, 2020, p. 270-273).

6. Rhet. Her., 12 ; Inv., I, 27.

7. Pour une analyse de cette tripartition, organisée selon l'utilité décroissante des narrations dans l'éloquence judiciaire, voir M. Formarier, « La narratio chez Cicéron doit-elle être brève pour persuader? », Interférences (http://journals.openedition.org/interferences/6007), n 10, 2018. La critique signale de façon suggestive que l'agrément (« delectatio ») peut déjà être une raison pour que l'orateur recoure au second type de narration.

8. In dialogum de partitione oratoria M. Tulii Ciceronis, commentarius, éd. Milan, Pacifico Da Ponte, 1569.

9. A.-É. Spica donne une vue d'ensemble du même type pour la description dans « Le traitement de la description dans les manuels de rhétorique à l'usage des élèves au $\mathrm{XVII}^{\mathrm{e}}$ siècle ", Pratiques : linguistique, littérature, didactique, $\mathrm{n}^{\circ}$ 109-110, 2001, p. 15-33.

10. Ch. Noille éclaire dans "Narratio / narration ?...", op. cit., p. 92-93, les types d'approche qui président à cette différence. Dans un cas la narration est intégrée dans le dispositif probatoire, «fonctionnalis[ée]»; de l'autre elle prend de l'autonomie, se trouve sortie de la «tension discursive ».

11. Rhétorique Françoise, éd. Paris, Saillant et Desaint, 1765, t. I, II, chap. 1, art. 2, «De la narration », p. 348-349. L'auteur précise : "Sans détruire la substance du fait, il [l'orateur] le présente sous des couleurs favorables : il insiste sur les circonstances qui lui sont avantageuses, et les met dans le plus beau jour : il adoucit celles qui seroient odieuses et choquantes. ».

12. La Rhétorique françoise, éd. Paris, Pierre le Petit, 1660, «De la narration », p. 267. Le passage traduit un vers en latin bien connu, attribué à Quintilien : "Quis, quid, ubi, quibus auxiliis, cur, quomodo, quando ".

13. La Rhétorique françoise, éd. Paris, Jacques Du Roure, 1662, p. 44.

14. La retorica, éd. Venise, Gabriel Giolito de Ferrari, 1559, VI, p. 441-447.

15. De eloquentice sacroe et humance parallela, éd. Paris, Sébastien Chappelet, 1619, VI, chap. 13, «De narratione. Narrationis divisio et usus », p. 222.

16. Ch. Noille rappelle dans « Narratio / narration ?...», op. cit., p. 92, que l'adjectif gnarus vient du verbe (g)nosco, qui signifie « savoir».

17. Voir la distinction faite par F. Revaz et J.-M. Adam dans L'Analyse des récits, Paris, Seuil, «Mémo ", 1996, p. 16-17, entre la cause et le motif: la cause est naturelle, tandis que le motif suppose une volonté humaine. On peut dire que la narration oratoire vise à dégager des motifs.

18. Rhet. Her., 14-16 ; Inv., I, 28-30 ; Inst., IV, 2, 31-59.

19. Voir Inv., I, 28 : « on omet non seulement ce qui peut nous nuire mais aussi ce qui n'est ni nuisible, ni utile» (traduction G. Achard). M. Formarier montre dans «La narratio chez Cicéron... ", op. cit., que la brièveté est le point sur lequel Cicéron évolue le plus dans la théorie de la narration qu'il développe du De inventione à l'Orator. Elle fait l'hypothèse que la réduction de la place qu'il lui accorde tient à une approche personnelle du théâtre et à sa pratique du barreau : il commente plus longuement un passage de l'Andrienne de Térence dans le De oratore que dans le De inventione et fait dans ses discours des narrations de plus en plus fournies en détails, où il introduit parfois de la véhémence.

20. Inst., IV, 2, 40-51. 
21. F. Goyet estime dans Le Regard rhétorique, Paris, Classiques Garnier, 2017, p. 257, que la notion de «vraisemblable ", marquée du rejet platonicien de la rhétorique, n'est pas très adaptée au discours oratoire. Il propose de parler plutôt de probable: l'orateur doit prouver sa thèse aux juges, la rendre validable par eux.

22. Rhetorique françoise, op. cit., p. 45.

23. Part., 31-32.

24. Part., 32. Jean Lecointe montre ici que c'est principalement du commentaire des Partitiones oratorice de Majoragius que se sont inspirés les artisans du « retour de la suavitas » au XVI e siècle.

25. Rhet. Her., 13 ; Inv., I, 27.

26. Voir à nouveau l'article "L'agrément des faits... » de J. Lecointe pour un lien entre les principes de la délectation chez Agricola et ceux du mythos chez Aristote et un rapprochement entre l'approche humaniste de la suavitas et les premières théories du suspens, propre à l'épopée et au roman grec, chez Vida et Amyot.

27. Voir M. Formarier, "La narratio chez Cicéron... », op. cit. : «À partir du De oratore, Cicéron définit donc le statut et la finalité de la narratio oratoire ainsi : il s'agit d'un récit visant à susciter et maintenir l'intérêt du public pour l'affaire dont il est question, au moyen de procédés mimétiques issus du théâtre (représentation morale et émotionnelle). »

28. Dans son développement sur la narration de L'arte oratoria secondo i modi della lingua volgare, éd. Venise, Giovanni Griffin, 1546, I, f. 13-13 vo , Francesco Sansovino mentionne des annales et des chroniques latines et italiennes.

29. Rhet. Her., 13, Inv., I, 27. La source en est a priori grecque. Sextus Empiricus attribue la distinction entre le mythos, l'istoria et le plasma à Asclépiade de Myrlée, qui a vécu vers l'an 200 av. J.-C. Voir le passage des Hypotyposes pyrrhoniennes cité par X. Riu dans «La place du vraisemblable dans la littérature grecque ", Pallas, $n^{\circ}$ 91, 2013, p. 49-60. Selon le rapport de Sextus Empiricus, Asclépiade identifie trois sortes d'histoires, qu'il définit et illustre par des genres, et subdivise encore l'istoria à partir des objets de celle-ci, à savoir des personnes, des lieux et des époques ou des actions.

30. Il y a toutefois des exceptions. Le Gras dans La Rhetorique Françoise, op. cit., II, 3, p. 111-114, reprend toutes les classifications des deux traités antiques au début de son chapitre sur la narration.

31. La Rhétorique françoise..., op. cit., p. 267. Sa catégorie du "prophetique » rappelle Quintilien, Inst., IV, 2, 3, sur l'exposé des faits à venir, "futurorum ", écarté comme oiseux : "forme de narration qui peut être réservée aux seuls devins [uaticinantibus]»; nous remercions Francis Goyet pour ce rapprochement.

32. Ibid., p. 266.

33. Rhetorique françoise, op.cit., p. 44. Il donne pour exemples de la première espèce «le ravissement de Proserpine dans les Verrines de Ciceron, et l'Histoire de la Berne dans les Lettres de Voiture ", donc un discours et une lettre privée.

34. De syllabarum quantitate epographice sex, éd. Pavie, Giacomo de Burgofranco, 1511, I, chap. 6, $\mathrm{f}^{\circ} 2 \mathrm{v}^{\circ}-3$; Poetica, éd. Paris, Josse Bade, 1520, f. A4.

35. Ch. Noille examine dans « Narratio / narration ?... ", op. cit., p. 93-96, le cas de la chrie active, par opposition à la chrie verbale, qui apprend à passer d'un sujet formulé en une phrase à un énoncé long intégrant des commentaires.

36. Inst., II, 4.

37. Aphthonii Sophistae Progymnasmata, éd. Cologne, Arnold Birckmann, 1562, «Narratio », f. 16 $\mathrm{v}^{\circ}-24$.

38. Candidatus Rhetoricoe, éd. Munich, Wagner Schell, 1664, « De Narratione », p. 288-330.

39. Candidatus Rhetorica, op.cit., V, «De Fabula», p.168-194 (L'Élève de rhétorique, op.cit., p. 256-285). 
40. De duplici copia verborum ac rerum, II, « De exemplo fabuloso », éd. B. I. Knott, dans Érasme, Opera omnia, Amsterdam, North-Holland, t. I-6, 1988, p. 234-240.

41. Dubois use aussi de la triade scolaire dans sa Poetica, parlant de genus historicum, genus oratorium et genus poeticum. Voir Poetica, op. cit., f. A4.

42. R. Baroni estime dans La Tension narrative. Suspense, curiosité, surprise, Paris, Seuil, 2007, p. 43-48, que les deux phénomènes sont présents dans tout récit. Il récuse l'idée de F. Revaz et J.M. Adam dans L'Analyse des récits, op. cit., p. 67-69, selon laquelle la mise en intrigue et la tension dramatique, se situant à deux plans différents, compositionnel et sémantique, sont indépendantes. Pour lui tension et intrigue sont en corrélation : tous les récits font attendre leur fin, aussi bien dans leur situation initiale que dans leur nœud.

43. Sansovino indique dans L'arte oratoria secondo i modi della lingua volgare, op. cit., I, f. 12, que l'orateur peut suivre un ordre «naturale » ou « artificiale », ce qui peut surprendre.

44. Du Roure, Rhetorique françoise, op. cit., p. 45.

45. Marmontel, Éléments de littérature, éd. Paris, Verdière, 1818, t. XIV, article "Narration ", p. 326-347 (éd. S. Le Ménahèze, Paris, Desjonquères, 2005, p. 755-766).

46. Inv., I, 30.

47. Quintilien envisage l'evidentia comme une qualité possible en s'affiliant à Cicéron, qui en fait un des ressorts de l'agrément narratif. Voir Inst., IV, 2, 63.

48. Ibid., IV, 2, 111-115.

49. Rhetoricorum libri V, éd. Bâle, Valentino Curio, 1522, I, « De Narratione », f. 14 v-15.

50. Voir Rhétorique Françoise, op. cit., t. I, II, chap. 1, art. 2, « De la narration », p. 361 : « si la cause est grande par son objet et par le nom des personnes qu'elle regarde ; si elle est variée par une multiplicité d'événemens divers; si elle est susceptible de sentimens de douleur, de commisération, d'indignation, de surprise, alors une narration froide et séche seroit tout-à-fait vicieuse. Elle doit être relevée par la noblesse du style, intéressante par les sentimens [...]. ».

51. Ibid., t. I, II, chap. 1, art. 2, « De la narration », p. 366.

52. L'Eloquence de la chaire ou la Rhetorique des predicateurs, éd. Paris, Académie des orateurs, 1665.

53. L'Eloquence de la chaire, et du barreau, éd. Paris, Denis Thierry, 1698, II, chap. 3, p. 137.

\section{AUTEUR}

PASCALE MOUNIER

Université Grenoble Alpes/CNRS - UMR 5316 Litt\&Arts 